\title{
PROPOSALS FOR SMALL STEPS TOWARD REPRODUCIBILITY OF SCIENCE EDUCATIONAL STUDIES
}

\author{
Andrej Šorgo \\ University of Maribor, Slovenia
}

It is evident that almost nobody is satisfied with contemporary (science) education, however, critique and proposed solutions to the perceived educational problems are sometimes established from opposite reasoning. Nowadays we can witness, for example, debates about position, role and effect of digital mobile technologies, social networks, and many other issues on behaviour and performance of students. Suggestions about their place in science education are on a scale between transforming education toward digital technologies and calls to prohibit, at least some of them, on the other end. Unfortunately enough argumentation too many times follows patterns recognized as 'The demon-haunted world'(Sagan, 1995) and a vocabulary and argumentation in line with Frankfurt's (2005) famous essay 'On bullshit'.

Optimally, argumentation for or against changes and follow up interventions should be empirically validated in a way, that facts are clearly separated from opinions. Common sense is that the crucial part in the process toward evidence-based interventions should be delegated to the researchers. However, it seems that they too many times forget to provide enough clues for their primary research to be understood and their findings to be verified, making their findings irreproducible. This does not mean that researchers are intentionally publishing falsified findings, however "Humans are remarkably good at self-deception." (Nuzzo, 2015, p. 182). Therefore, reproducibility should be the key quality of empirical research.

There are many good reasons, why published studies should not be regarded as a one-time event but as a brick in a cathedral of knowledge. The sentence, "No research paper can ever be considered to be the final word, but there are too many that do not stand up to further study" cited from the collection of articles published under an umbrella title "Challenges in irreproducible research" (Nature, n. d.) points to the problem. As an example, we can once again consider educational technologies, which had substantially changed science education and our private lives in last decades, as well. Therefore, findings and conclusions presented in pioneering papers are not necessarily valid anymore, however, methodology, if properly presented, can allow repetition of the study. The same is true for comparative studies, because something valid for one culture or population is not necessary valid under different conditions. Additionally, statistics is a very vivid research field and with greater computer power allows procedures which were not available at the time of the study or are out of the statistical horizons of a researcher. Repetition is, however, not possible without access to the primary data.

I would like to present a short list of simple procedures that can transfer published papers from gravel in a pathway toward to a keystone in an arch of knowledge. As a reader and potential user of your valuable research, I would greatly appreciate that your papers follow at least majority of the listed:

- Clearly concept your research questions in a way that they can be reused by yourself and the others in follow up studies.

- Hypotheses should not be presented as educated guesses about expected outcomes, but should be regarded as research questions translated in equations presented by words and/or symbols, to be tested by one or the other test.

- It should be known who or what constitute your population (e.g. number of all secondary students of particular age in a country) by providing exact population numbers and qualitative and quantitative description of traits and population strata considered in a study. 
ISSN 1648-3898/Print/

ISSN 2538-7138/Online/

- Describe in enough details how well your sample represents your population, and what are sources of possible bias.

- Provide enough details of sampling procedure and data collection. Provide assumptions what can be potential and actual sources of bias.

- In some or the other way provide in appendix, supporting materials and/or link to the permanent page access to the research instruments.

- Provide permanent access to your primary data even after you have achieved your degree, have moved to another institution or went retired.

- Report statistical parameters in enough details to allow inclusion of your findings in secondary studies. At least what you should provide in empirical educational studies is listed below:

- For each described and tested group provide the exact sample size ( $n$ ) as a discrete number.

- Provide units for assessment for each condition and description of all applied covariates.

- Provide indication of any assumption connected to your variables (normality, skewness, and similar) and corrections (exclusion of outliers, handling with missing data, transformations, and similar).

- Ensure that the error bars are defined and state explicitly what they represent throughout the figures (e.g. confidence intervals, standard deviation, standard error, range, etc.).

- Provide a full descriptive statistics of each variable by inclusion of frequencies, measures of central tendency (means, mode), variation (e.g. standard deviation) or associated estimates of uncertainty (e.g. confidence intervals).

- Provide names of all applied statistical test(s) and state whether they are one- or two-sided.

- Provide outcomes of the test statistic by reporting exact numbers (e.g. $F, U, Z, t, r$ ) with confidence intervals, effect sizes, degrees of freedom and $p$ values.

- Report exact $p$-values even if they are statistically non-significant whenever relevant.

- When applicable present multilevel and complex models as graphics.

- Provide estimates of effect sizes (e.g. Cohen's $d$, Pearson's $r$ ) with confidence intervals and reference to the calculations.

- When reporting exploratory factorial analyses provide information about the initial set of items, excluded items, and reasoning for retaining factors (parallel analysis), together with estimates of reliability.

- $\quad$ Order items in tables in some logical order.

- Conclusions should be answers to the research questions.

- Provide assumptions of limitations of the study.

Following this list of suggestions cannot guarantee that your paper is published, because nothing can replace evidence of comprehensive background research, creativity in producing attractive ideas, and formal reasoning in defending them. However, you should not be surprised if a number of reviewer's recommendations will drop, and the number of downloads and papers citing your published work is going to climb.

\section{References}

Frankfurt, H. G. (2005). On bullshit. Princeton, New Jersey. Princeton University Press.

Nature (n. d). Challenges in irreproducible research. Retrieved from https://www.nature.com/collections/prbfkwmwvz.

Nuzzo, R. (2015). How scientists fool themselves-and how they can stop. Nature News, 526 (7572), 182.

Sagan, C. (1995). The demon-haunted world: Science as a candle in the dark. New York. Random House.

Andrej Šorgo 